University of Nebraska - Lincoln

DigitalCommons@University of Nebraska - Lincoln

Biological Systems Engineering: Papers and

Publications

Biological Systems Engineering

$1-2010$

\title{
Modeling Changes in Biomass Composition During Microwave- Based Alkali Pretreatment of Switchgrass
}

Deepak R. Keshwani

University of Nebraska-Lincoln, dkeshwani2@unl.edu

Jay J. Cheng

North Carolina State University, jay_cheng@ncsu.edu

Follow this and additional works at: https://digitalcommons.unl.edu/biosysengfacpub

Part of the Biological Engineering Commons

Keshwani, Deepak R. and Cheng, Jay J., "Modeling Changes in Biomass Composition During MicrowaveBased Alkali Pretreatment of Switchgrass" (2010). Biological Systems Engineering: Papers and Publications. 177.

https://digitalcommons.unl.edu/biosysengfacpub/177

This Article is brought to you for free and open access by the Biological Systems Engineering at DigitalCommons@University of Nebraska - Lincoln. It has been accepted for inclusion in Biological Systems Engineering: Papers and Publications by an authorized administrator of DigitalCommons@University of Nebraska Lincoln. 


\title{
Modeling Changes in Biomass Composition During Microwave-Based Alkali Pretreatment of Switchgrass
}

\author{
Deepak R. Keshwani \\ Department of Biological Systems Engineering, University of Nebraska-Lincoln, Lincoln, Nebraska 68583-0726
}

\begin{abstract}
Jay J. Cheng
Department of Biological and Agricultural Engineering, North Carolina State University, Raleigh, North Carolina 27695-7625 [Corresponding author - tel 919 515-6733, fax 919 515-7760, e-mail jay_cheng@ncsu.edu ]
\end{abstract}

\begin{abstract}
This study used two different approaches to model changes in biomass composition during microwavebased pretreatment of switchgrass: kinetic modeling using a time-dependent rate coefficient, and a Mamdani-type fuzzy inference system. In both modeling approaches, the dielectric loss tangent of the alkali reagent and pretreatment time were used as predictors for changes in amounts of lignin, cellulose, and xylan during the pretreatment. Training and testing data sets for development and validation of the models were obtained from pretreatment experiments conducted using 1-3\% w/v NaOH (sodium hydroxide) and pretreatment times ranging from 5 to 20 min. The kinetic modeling approach for lignin and xylan gave comparable results for training and testing data sets, and the differences between the predictions and experimental values were within $2 \%$. The kinetic modeling approach for cellulose was not as effective, and the differences were within 5- 7\%. The time-dependent rate coefficients of the kinetic models estimated from experimental data were consistent with the heterogeneity of individual biomass components. The Mamdanitype fuzzy inference was shown to be an effective approach to model the pretreatment process and yielded predictions with less than $2 \%$ deviation from the experimental values for lignin and with less than $3 \%$ deviation from the experimental values for cellulose and xylan. The entropies of the fuzzy outputs from the Mamdani-type fuzzy inference system were calculated to quantify the uncertainty associated with the predictions. Results indicate that there is no significant difference between the entropies associated with the predictions for lignin, cellulose, and xylan. It is anticipated that these models could be used in process simulations of bioethanol production from lignocellulosic materials.
\end{abstract}

Keywords: kinetic modeling; fuzzy modeling; microwave pretreatment; switchgrass; bioethanol

\section{Introduction}

Lignocellulosic materials such as agricultural residues, forestry residues, herbaceous energy crops, and woody biomass are regarded as potential long-term alternative feedstock for bioethanol production. Switchgrass (Panicum virgatum L.), a promising lignocellulosic feedstock, is a warm-season perennial grass native to North America. It exhibits high biomass yields across a wide geographic range, has low water and nutritional requirements and is suitable for marginal quality land (McLaughlin, 1993). Yields of 7-15 dry tons per acre have been reported in the literature (Parrish and Fike, 2005; Sanderson et al., 1996; Thomason et al., 2004). The environmental benefits associated with switchgrass include the potential for carbon sequestration, nutrient recovery from run-off, soil remediation, and provision of habitats for grassland birds (Dunn et al., 1993; Keshwani and Cheng, 2009).

As is the case with corn and other starch-containing materials, bioethanol production from lignocellulosic materials involves hydrolysis of carbohydrates in the biomass into simple sugars that are fermented into ethanol. However, the nature of lignocellulosic materials reduces the efficiency of hydrolysis of structural carbohydrates in the raw biomass. Lignocellulosic materials consist of cellulose, hemicellulose, and lignin in a complex crystalline structure. This complex structure reduces accessibility of hydrolytic enzymes to the carbohydrates. As a result, a pretreatment step is required to improve efficiency of hydrolytic enzymes. The goals of pretreatment are to remove lignin and/or hemicellulose, reduce crystallinity of cellulose, and increase overall porosity of the biomass. A range of biological, chemical, physical, and physicochemical pretreatment methods have been explored. Sun and Cheng (2002) and Mosier et al. (2005) have summarized the modes of actions, and the advantages and disadvantages associated with different pretreatment methods. 
The use of microwave radiation is a promising approach to pretreatment that utilizes thermal and non-thermal effects caused by microwaves in aqueous environments. Several studies have reported enhanced sugar yields as a result of microwave-based alkali pretreatment of lignocellulosic materials. For example, Zhu et al. (2005) reported a glucose yield of $65 \%$ and total carbohydrate conversion of $78 \%$ from rice straw, $\mathrm{Hu}$ and Wen (2008) reported 70-90\% sugar yields from switchgrass, and Keshwani et al. (2007) reported $80-85 \%$ sugar yields from switchgrass.

The vibration of polar molecules and movement of ions in response to microwave radiation results in the generation of heat and extensive collisions. These thermal and non-thermal effects can accelerate chemical, biological, and physical processes (Sridar, 1998). The interaction of microwaves with any material depends on its dielectric properties: dielectric constant and dielectric loss factor. The dielectric constant is a measure of the ability of a material to store electromagnetic energy and the dielectric loss factor is a measure of the ability of a material to convert electromagnetic energy into heat (Kumar et al., 2007). Loss tangent, which is a ratio of the dielectric loss factor to the dielectric constant, is a parameter used to describe the overall efficiency of a material to utilize energy from microwave radiation (Nelson and Datta, 2001).

Lignin acts as a barrier to the enzymes and is known to irreversibly adsorb cellulase enzyme molecules (Converse et al., 1990; Lee et al., 1994). Therefore, the removal of lignin during alkali pretreatment helps to improve the efficiency of enzymatic hydrolysis. The separated lignin can be recovered from the pretreatment liquor and used as boiler-fuel for energy production. In the process of removing lignin, carbohydrate degradation into the pretreatment liquor can also occur. This can significantly reduce the amount of fermentable sugars available for ethanol production. Hence, the ability to predict changes in biomass composition during pretreatment is an important component of overall process simulations to evaluate the economics of bioethanol production from lignocellulosic feedstock. However, modeling the effects of pretreatment processes can be challenging because of the inherent variability and heterogeneity in the structure of lignocellulosic biomass. To date, there has been no published work that attempts to model the effects of microwave-based pretreatment.

A review of the literature indicates that most efforts to model pretreatment processes have focused on dilute acid pretreatment. Jacobsen and Wyman (2000) reviewed the different approaches to model the hydrolysis of carbohydrates during dilute acid pretreatment. Saeman (1945) described the acid hydrolysis of cellulose into glucose monomers and eventual degradation products using two consecutive first-order reactions. This model was subsequently improved to account for amorphous and crystalline regions in cellulose and potential reversible reactions between monomers and oligomers (Conner et al., 1985). A biphasic approach is commonly used to describe the dis- solution kinetics of fast reacting and slow reacting components of xylan during dilute acid pretreatment (Conner, 1984). A common theme among all these kinetic models is a first-order dependence on the biomass component.

Another approach to modeling the effects of pretreatments was proposed by Overend and Chornet (1987) who developed a severity factor to predict xylan solubilization as a function of time and temperature in hydrothermal processes. Chum et al. (1990) incorporated the effect of chemical concentration and proposed a modified severity factor. However, this modified severity factor has seen only limited applications in dilute acid pretreatment (Ramos, 2003). A notable exception is Silverstein et al. (2007) who used this factor to predict delignification during alkali pretreatment of lignocelluloses. Most models of delignification have been in the context of pulping processes and include a generalized severity parameter (Montane et al., 1994), single phase kinetics (Kerr, 1970), multiple phase kinetics (Bogren et al., 2007), combined diffusion and kinetics (Gustafson et al., 1983), and the superposition of three first-order sub-processes representing distinct fractions of lignin (Chiang et al., 1990).

The objective of this study was to develop models to predict changes in biomass composition during microwavebased alkali pretreatment of switchgrass. Two different modeling approaches were used: kinetic modeling using a time-dependent rate coefficient and a Mamdanitype fuzzy inference system. These approaches were chosen as they represent two different ways of dealing with the inherent variability and heterogeneity in the structure of lignocellulosic biomass. While the kinetic modeling approach was adapted from previously published work on pulping kinetics by Dang and Nguyen (2006, 2007), the fuzzy-logic-based Mamdani inference system was applied for the first time to model a pretreatment process. In both approaches, the dielectric loss tangent of the alkali reagent and pretreatment time were used to predict the amount of biomass components remaining in pretreated switchgrass relative to amounts present in untreated switchgrass. The inputs for the models are expressed as $D$ (loss tangent) and $t$ (pretreatment time). The outputs for the models are expressed as $L / L_{i}$ (for lignin), $C / C_{i}$ (for cellulose), and $X / X_{i}$ (for xylan), where $L, C$, and $X$ represent the amount of the biomass components present in switchgrass during the course of the pretreatment and $L_{i}, C_{i}$, and $X_{i}$ represent the amounts present initially in untreated switchgrass. While most pretreatment models typically use concentration of the chemical reagent as a predictor, in this study the dielectric loss tangent was chosen as a substitute for alkali concentration to model microwave-based pretreatment. Prior work has established that the dielectric loss tangent has a strong correlation with alkali concentration and pretreatment severity (Keshwani and Cheng, 2008). It is anticipated that the use of dielectric properties instead of concentration would enable the models to be easily adapted for a different alkali reagent. 


\section{Modeling Aspects}

\section{Kinetic Modeling Approach}

The general form of the kinetic model used in this study is based on previously published work by Dang and Nguyen (2006, 2007), who proposed fractal-like kinetics to describe delignification and carbohydrate losses of lignocellulosic materials. Based on heterogeneous mass transfer considerations (Avrami, 1940) and the power law of the growth of reactive sites in solid-state decomposition reactions (Bamford and Tipper, 1980), Dang and Nguyen $(2006,2007)$ derived the following expression relating the amount of a component (B) in lignocellulosic biomass to a time-dependent rate coefficient via a first-order-differential equation:

$$
-\frac{\mathrm{d} B}{\mathrm{~d} t}=\left(k n t^{n-1}\right) B
$$

$B$ is the amount of the component (lignin, cellulose, or xylan) present in the untreated biomass, and $k$ and $n$ are constants that are based on the nature of the heterogeneous material and the reaction involved. Equation (1) differs from commonly used first-order-kinetic models for delignification and carbohydrate solubilization in the fact that the rate coefficient $\left(k n t^{n-1}\right)$ is time dependent to reflect the heterogeneity in lignocellulosic materials (Montane et al., 1994). Previous studies typically introduce the effect of concentration into Equation (1). However, in this instance, the dimensionless dielectric loss tangent of the alkali reagent $(D)$ is introduced in Equation (1) to obtain the general form of the model:

$$
-\frac{\mathrm{d} B}{\mathrm{~d} t}=\left(k n t^{n-1}\right) D^{b} B
$$

At this point, an assumption is made that the alkali concentration remains relatively constant during the course of the short pretreatment times $(\leq 20 \mathrm{~min})$ and consequently the rate of change of the dielectric constant with respect to time is assumed to be zero. This assumption is commonly used to determine pulping kinetics and is considered valid when the initial effective alkali loading (per gram of biomass) is high. If the dielectric loss tangent is assumed constant during the course of the pretreatment, Equation (2) can be integrated and linearized as shown in Equation (3). The linearization enabled the use of a regression routine with experimental data to determine the model parameters $(k, n$, and $b)$.

$$
\ln \left[-\ln \left(\frac{B}{B_{\mathrm{i}}}\right)\right]=\ln (k)+b \ln (D)+n \ln (t)
$$

\section{Mamdani-Type Fuzzy Inference System}

The second modeling approach used is a Mamdanitype fuzzy inference system, in which a set of user-defined rules maps the input space to the output space. The inference system was developed using the fuzzy logic toolbox in MATLAB (Version 2007a, Mathworks, Cambridge, MA). The Mamdani-type inference system is an example of a fuzzy relational model where each rule is represented by an IF- THEN relationship between inputs and outputs. It is also called a linguistic model because the antecedents (inputs) and the consequents (outputs) are represented by fuzzy sets called membership functions (Babuska, 1998). As a result, it is common to see membership function in such models labeled in linguistic terms such as low, medium, and high that represent specific regions in each dimension of the input and output data space.

In general, fuzzy-logic-based models are used to deal with non-random uncertainty in complex systems and experimental data (Ross, 1995). This uncertainty is typically reflected by overlapping the membership functions in each dimension of the input and output space. The complex structure of lignocellulosic materials and the inherent variability in biomass characteristics prompted the use of such an approach. Examples of recent applications of fuzzylogic- based modeling in different areas include prediction of surface properties of gum and proteins (Samhouri et al., 2009), soil quality assessment (Torbert et al., 2008), and modeling of skin permeability (Keshwani et al., 2005, 2008; Pannier et al., 2003). To date, this approach has not been applied to model the pretreatment of lignocellulosic biomass.

There are three components to a Mamdani-type fuzzy inference system: input membership functions, output membership functions, and the rule-base. The rule-base is used to predict outputs as a fuzzy set, which can be converted into a crisp estimate via defuzzification routines. Depending on the values of the inputs, multiple rules can be activated and the outputs from each rule are aggregated to form a fuzzy set representing the predicted output. The output fuzzy set is then defuzzified to yield a crisp value which can be compared to experimental values. An example of a simple Mamdani-type inference system is shown in Figure 1, where a rule-base consisting of four rules is used to predict one output from two inputs. The inputs and output space are each defined by two membership functions (low and high) and the rules map the inputs to the output. For example, Rule 2 states that if Input 1 is low and Input 2 is high, then the corresponding output is low (Figure 1).

Babuska (1998) and Ross (1995) provide a detailed description of different types of membership functions and defuzzification routines that are commonly used. In a Mamdani-type inference system, these membership functions and rules are defined by the modeler based on knowledge of the system or process being modeled. The knowledge is typically gained from expert opinion and/ or observations from experimental data. In this study, the number of membership functions for the model's inputs and their location in the respective data space were based on the levels of these factors in the experimental design 


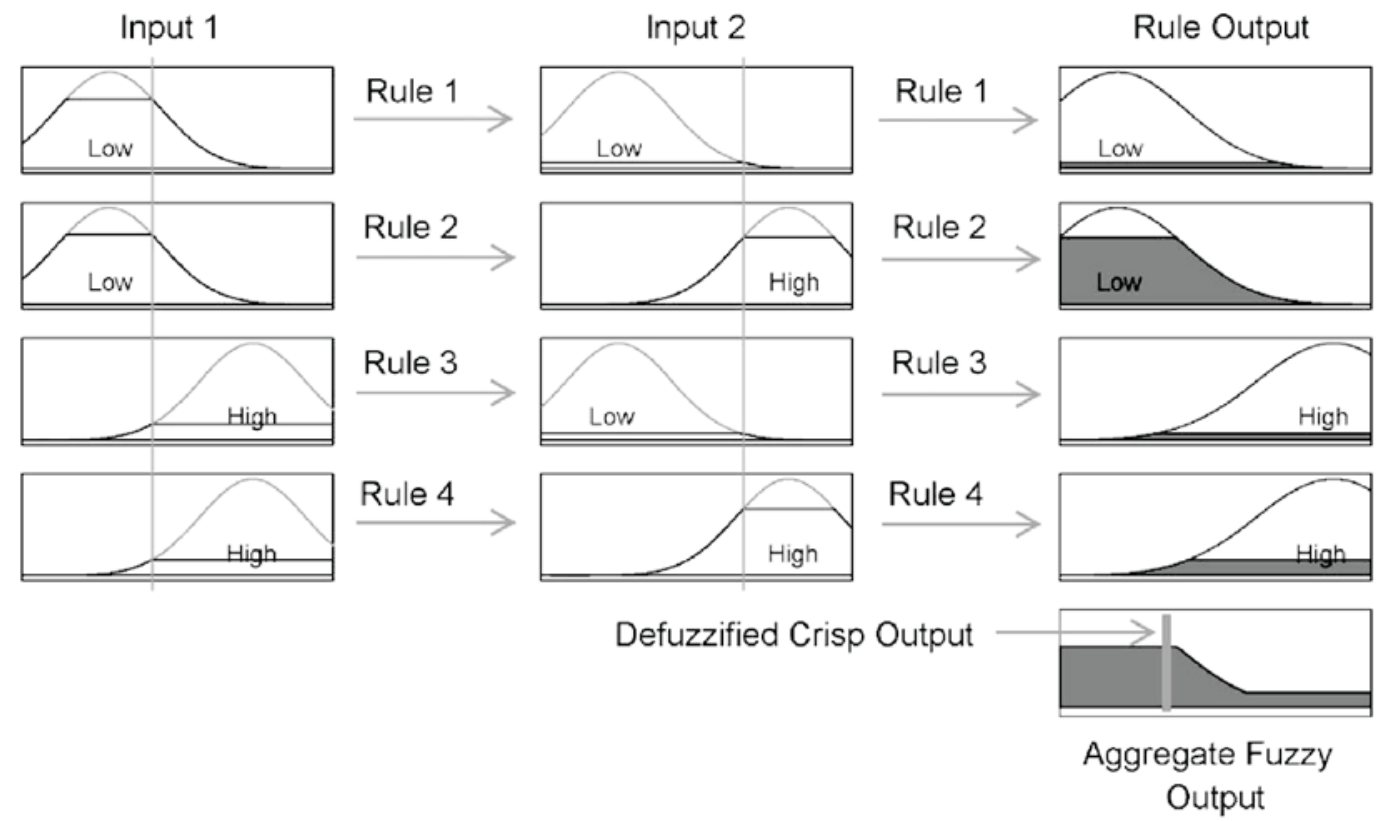

Figure 1. Example of a simple Mamdani-type fuzzy inference system that uses four rules to predict a single output from two inputs.

used to generate the training data set. The number of membership functions for the outputs and their location were determined by clustering experimental data. First, subtractive clustering was used to estimate the number of membership functions for each output. These estimates were then used to cluster the output data using the Fuzzy C-means algorithm (FCM). Compared to subtractive clustering, FCM is a relatively more sophisticated clustering routine that maximizes distances between cluster centers and minimizes distance between each point and the cluster centers while maintaining the possibility that a data point can belong to more than one cluster.

Once the membership functions were determined, the rule-base was developed manually using observations from the training data set. Hence, the Mamdani-type inference system developed in this study can be considered a pseudodata driven model since experimental data was only used to identify the location of membership functions and as a knowledge source in the manual development of rules. Modeling using a Mamdani-type inference system has several advantages. Multiple outputs can be predicted using the same inference system, rules with same input conditions can infer different outputs with varying weights to reflect uncertainty in the system, and membership functions and rules can be easily modified to reflect changes in the system.

\section{Materials and Methods}

\section{Biomass}

Switchgrass (Performer cultivar) was obtained from North Carolina State University's Central Crops Re- search Station in Clayton, North Carolina. The feedstock was oven-dried, ground to pass a $2 \mathrm{~mm}$ sieve in a Thomas Wiley Laboratory Mill (Model No. 4) and stored in sealed plastic bags at room temperature until use for pretreatment.

\section{Pretreatment}

Microwave-based alkali pretreatment of switchgrass using $\mathrm{NaOH}$ as an alkali reagent was carried out in a general-purpose laboratory microwave oven (Panasonic Corporation, model NN-S954). All pretreatments in this study were carried out at a power level of 250 Wthat was identified from preliminary experiments. For each pretreatment, slurry consisting of $5 \mathrm{~g}$ (dry basis) of the biomass immersed in dilute $\mathrm{NaOH}$ solutions (solid to liquid ratio of 1:10) was exposed to microwave radiation for the desired pretreatment time. Following the pretreatment, the resulting biomass was washed with $200 \mathrm{~mL}$ of deionized water to remove excess chemicals and/or other pretreatment byproducts. Moisture contents of the pretreated biomass were measured after washing and samples were stored in a sealed plastic bag at $4^{\circ} \mathrm{C}$ for composition analysis. Experiments were conducted in triplicate at $\mathrm{NaOH}$ concentrations ranging from 1 to $3 \%$ and pretreatment times ranging from 5 to $20 \mathrm{~min}$. Two-thirds of the experimental data (12 experimental conditions) was used as a training data set for model development and the remaining one-third (6 experimental conditions) was used as a testing data set for model validation.

\section{Biomass Composition Analysis}

Structural carbohydrates and lignin content in untreated and pretreated switchgrass were measured by a 
two-step acid hydrolysis procedure as per a standard protocol from the National Renewable Energy Laboratory (Sluiter et al., 2007). Cellulose and xylan content was calculated from glucose and xylose using anhydro corrections of 0.90 and 0.88 , respectively. Monomeric sugars were measured with an HPLC using a refractive index detector and a Bio-Rad Aminex HPX-87P column tailored for separation of monosaccharides derived from lignocellulosic materials. The column was operated at a flow rate of $0.6 \mathrm{~mL} / \mathrm{min}$ and at a temperature of $80^{\circ} \mathrm{C}$ using HPLC grade water as the mobile phase. The injection volume for the HPLC samples was $10 \mathrm{~mL}$ and the sample-run time was $35 \mathrm{~min}$ with a postrun time of $25 \mathrm{~min}$.

\section{Measurement of Dielectric Properties}

Dielectric properties of $\mathrm{NaOH}$ were measured using an open-ended coaxial dielectric probe (Model HP 85070B, Agilent Technologies, Palo Alto, CA) connected to a network analyzer (Model HP 8753C, Agilent Technologies) based on a previously reported procedure (Kumar et al., 2007). All measurements were made in triplicate. The solutions were placed in a cylindrical vessel fitted with a dielectric probe and temperature probe and sealed using a tri-clamp fitting. The vessel was heated to desired temperatures using an oil bath (Model RTE111, Neslan Instruments Inc., Newington, NH). The network analyzer was programmed to record dielectric properties for frequencies ranging from 200 to 3,000 MHz when a desired temperature was reached.

\section{Analysis of Models}

The performance of the models was evaluated using $R^{2}$ values from the regression line fitting experimental values from training and testing data to values predicted by the model, and root mean square errors (RMSE) associated with the predictions of training and testing data. The Shapiro-Wilk test of normality was applied to prediction residuals obtained when the models were applied to training and testing data. This was done to confirm that the models are not significantly over or under-predicting the outputs and that the residuals are samples from a normal distribution. This test has been determined to be robust for small datasets (Shapiro and Wilk, 1965). The null hypothesis that the residuals are normally distributed is not rejected if the Shapiro-Wilk test statistic is close to 1 and the associated $P$-value is greater than a predefined alpha level (0.05).

A sensitivity analysis on the parameters of the kinetic models was conducted by calculating absolute value of the relative sensitivity index at 10 randomly chosen data points. The relative sensitivity index $\left(R_{\mathrm{S}}\right)$ is defined as the ratio of the relative normalized change in a model's output to the relative normalized change of a specific parameter (Turanyi and Rabitz, 2000). $R_{\mathrm{S}}$ can be calculated using Equation (4), in which $O$ refers to the model output and $P$ refers to the parameter of interest.

$$
R_{\mathrm{S}}=\frac{P}{O} \frac{\Delta O}{\Delta P}
$$

While the predicted output of the Mamdani-type inference system can be defuzzified to yield a crisp value for comparison with experimental data, the uncertainty associated with the aggregate fuzzy output can be quantified by calculating its entropy. The entropy of a fuzzy set $(E)$ can be quantified in terms of a lack of distinction between a fuzzy set and its complement (Hung, 2003) and is calculated as follows:

$$
E=1-\frac{1}{N} \sum_{i=1}^{N}\left|2 \mu_{i}-1\right|
$$

In Equation (5), $N$ is the number of points that define the set and $\mu_{i}$ is the membership value of each point that defines the set. The entropy value can range from 0 to 1 depending on the degree of fuzziness associated with the set. A crisp value (not a fuzzy set) will have an entropy of 0 and a fuzzy set in which all points have the same membership value will have an entropy of 1 . The entropies of the fuzzy outputs from the Mamdani-type inference system was calculated using Equation (5) to compare the level of uncertainty associated with the predictions for lignin, cellulose, and xylan.

\section{Results and Discussion}

\section{Kinetic Model}

The parameters $(k, n$, and $b)$ of the models for lignin, cellulose, and xylan estimated from the training data set are shown in Table I. The $P$-values in each case were very low $\left(<10^{-4}\right)$ indicating a high degree of confidence in the estimates of these parameters. The results of the sensitivity analysis (Table II) indicate that the models for all biomass components were most sensitive to changes in the values of $k$ and $n$. These findings are consistent with those of Dang and Nguyen (2006) who used this modeling approach to predict changes in lignin content during alkaline pulping and reported that model predictions were highly sensitive to a change in the value of $n$ but not to a change in the value of $b$.

Of particular interest is the value of $n$, which dictates the time dependency of the overall rate coefficient for each biomass component. Figure 2 shows the behavior of the overall rate coefficients $\left(k n n^{n-1}\right)$ over the course of the pretreatment. If the value of $n$ is close to 1 (observed for cellulose), then the rate coefficient does not vary significantly over time. As the value of $n$ moves away from 1 (xylan followed by lignin), the rate coefficient will exhibit significant variability over time. According to Montane et al. (1994), the use of time-dependent rate coeffi- 
Table I. Parameter values for the kinetic modeling approach (Equation 1) for each biomass component.

\begin{tabular}{lrrr}
\hline Model & $k$ & $n$ & $b$ \\
\hline Lignin $\left(L / L_{\mathrm{i}}\right)$ & 0.499 & 0.188 & 0.31 \\
Cellulose $\left(C / C_{\mathrm{i}}\right)$ & 0.011 & 0.874 & 1.03 \\
Xylan $\left(X / X_{i}\right)$ & 0.073 & 0.815 & 0.49 \\
\hline
\end{tabular}

Table II. Absolute values of relative sensitivity indices (RS) for $\pm 10 \%$ change in estimated values for kinetic model parameters shown in Table I.

\begin{tabular}{|c|c|c|c|c|c|c|}
\hline \multirow[b]{2}{*}{ Model } & \multicolumn{2}{|r|}{$k$} & \multicolumn{2}{|r|}{$n$} & \multicolumn{2}{|c|}{$b$} \\
\hline & $+10 \%$ & $-10 \%$ & $+10 \%$ & $-10 \%$ & $+10 \%$ & $-10 \%$ \\
\hline Lignin $\left(L / L_{\mathrm{i}}\right)$ & 0.413 & 0.428 & 0.497 & 0.469 & 0.074 & 0.074 \\
\hline Cellulose $\left(C / C_{\mathrm{i}}\right)$ & 0.342 & 0.326 & 0.387 & 0.473 & 0.056 & 0.059 \\
\hline Xylan $\left(X / X_{\mathrm{i}}\right)$ & 0.315 & 0.304 & 0.337 & 0.365 & 0.106 & 0.107 \\
\hline
\end{tabular}

cients reflects a physical situation in which the reacting substrate is composed of many chemical species of similar nature but with different reaction rates. Hence, the estimated values of $\mathrm{n}$ should be consistent with the nature of the biomass components. Cellulose is a homopolymer, consisting of only glucose and hence the rate coefficient for cellulose loss should not vary over time. Although xylan is primarily made up of xylose, it is associated with other minor carbohydrates and acetyl groups resulting in a random interlinked structure. Therefore, the variability in its rate coefficient shown in Figure 2 is reasonable. Lignin is a complex polymer consisting of numerous types of phenolic sub-structures that are interlinked via random ether and carbon-carbon linkages. Hence, it is reasonable to expect that the rate coefficient would vary significantly over time.

The $R^{2}$ and RMSE values for the training and testing data sets for the kinetic models for lignin, cellulose, and xylan are shown in Table III. The training- $R^{2}$ values for lignin, cellulose, and xylan were $0.95,0.87$, and 0.98, respectively, and the corresponding training-RMSE values were $0.016,0.051$, and 0.019 . Note that the RMSE values for cellulose are significantly higher than those for lignin and xylan. The testing- $R^{2}$ and testing-RMSE values for lignin and xylan obtained for the testing data set are comparable to those obtained for the training data set. However, for cellulose, the $R^{2}$ reduces to 0.81 and RMSE increases to 0.067 for the testing data set. For all biomass components, the Shapiro-Wilk test statistic for the prediction residuals was $>0.9$ and was significant at an alpha level of 0.05 .

These results indicate that the kinetic modeling approach provides a good representation of the changes in lignin and xylan content with predictions that had less than $2 \%$ deviations from the experimental values. However, the modeling approach is less accurate for cellulose. Dang and Nguyen (2006) reported similar findings
Table III. $R^{2}$ and RMSE values for the kinetic and fuzzy models applied to training and testing data sets.

\begin{tabular}{lcccc}
\hline & \multicolumn{2}{c}{ Training data set } & \multicolumn{2}{c}{ Testing data set } \\
Modeling approach & $R^{2}$ & RMSE & $R^{2}$ & RMSE \\
\hline Kinetic modeling & & & & \\
$\quad$ Lignin $\left(L / L_{\mathrm{i}}\right)$ & 0.95 & 0.016 & 0.95 & 0.018 \\
$\quad$ Cellulose $\left(C / C_{\mathrm{i}}\right)$ & 0.87 & 0.051 & 0.81 & 0.067 \\
$\quad$ Xylan $\left(X / X_{\mathrm{i}}\right)$ & 0.98 & 0.019 & 0.98 & 0.022 \\
Fuzzy inference system & & & & \\
$\quad$ Lignin $\left(L / L_{\mathrm{i}}\right)$ & 0.95 & 0.014 & 0.94 & 0.013 \\
$\quad$ Cellulose $\left(C / C_{i}\right)$ & 0.96 & 0.021 & 0.87 & 0.028 \\
$\quad$ Xylan $\left(X / X_{\mathrm{i}}\right)$ & 0.98 & 0.018 & 0.95 & 0.022 \\
\hline
\end{tabular}

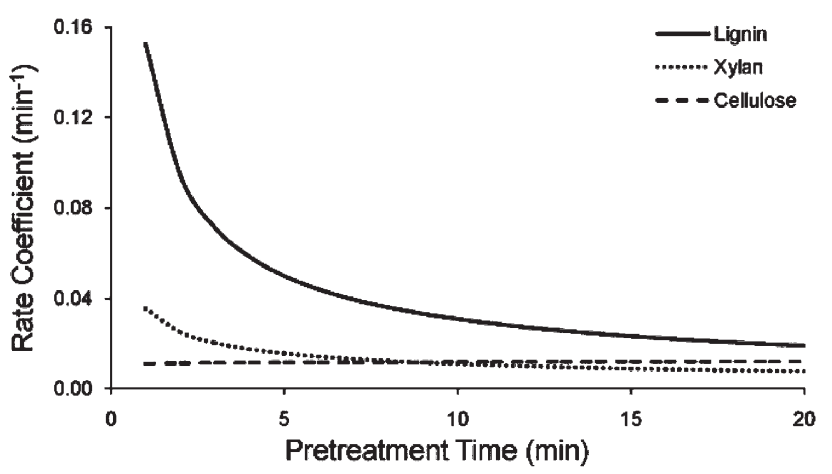

Figure 2. Behavior of rate coefficients for the kinetic models over the course of the pretreatment.

when this modeling approach was used to model the kinetics of alkaline pulping of hemp wood core. The study reported $R^{2}$ values of 0.95 for delignification, 0.97 for $\mathrm{xy}$ lan loss, and 0.80 for cellulose loss and noted that variation in the ratio of crystalline and non-crystalline cellulose components in test samples could affect the accuracy of the model.

\section{Mamdani-Type Fuzzy Inference System}

The Mamdani-type fuzzy inference system was developed using the fuzzy logic toolbox in MATLAB. The membership functions for the inputs (shown in Figure 3) were based on their levels in the experimental design used to collect the training data set. The membership functions for the outputs (shown in Figure 4) were derived using subtractive and FCM clustering of the training data set. Using information from the training data set, a rule-base consisting of 32 rules was developed to define the relationships between the input and output membership functions. A verbal representation of these rules is shown in Table IV.

The inference system was applied to the training and testing data sets and the corresponding $R^{2}$ and RMSE values are shown in Table III. The training- $R^{2}$ values for lignin, cellulose, and xylan were $0.95,0.96$, and 0.98 , respectively, and the corresponding training-RMSE values were $0.014,0.021$, and 0.018 . For all biomass components, the 

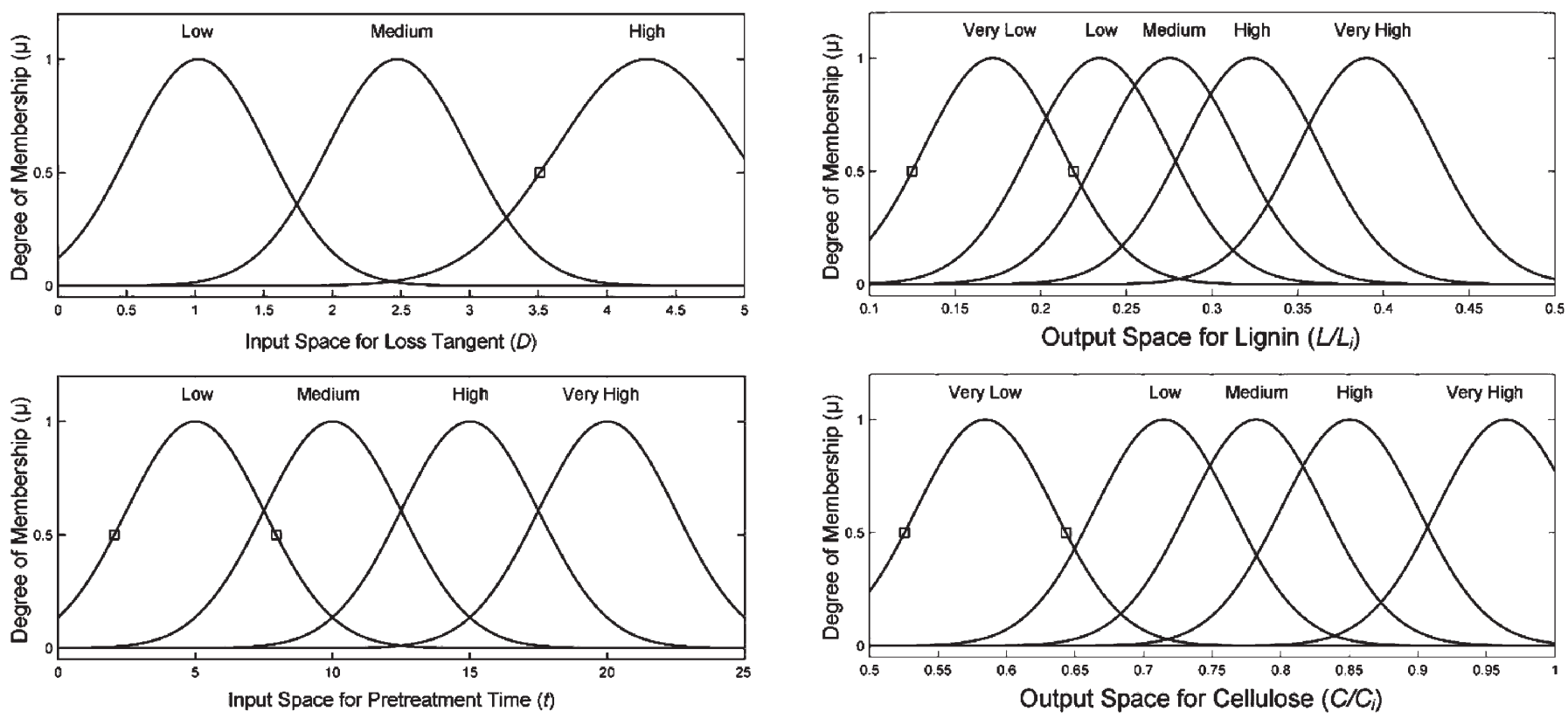

Figure 3. Membership functions for the inputs of the Mamdani-type fuzzy inference system.

RMSE values for the testing data set are comparable to those obtained for the training data set and the ShapiroWilk test statistic for the prediction residuals was $>0.9$ and was significant at an alpha level of 0.05 .

From Table III, it should be noted that the RMSE value for the prediction of cellulose content for the testing data set using the fuzzy inference system is considerably lower than that obtained for the kinetic model ( 0.028 vs. 0.067$)$. It could be inferred that the uncertainty associated with the crystalline/amorphous nature of cellulose is being captured by using overlapping fuzzy sets to define the input and output space. The overall consistency in predictions with training and testing data sets for the Mamdani-type inference system is not surprising since the relationship between the outputs and inputs (i.e., the rule-base) was manually developed and is therefore not prone to overfitting caused by data regression. It should be noted that the development of the inference system can be an iterative process based on the extent of familiarity with the system being modeled.

The entropy is indicative of the uncertainty associated with the predicted fuzzy output of a Mamdani-type fuzzy inference system. The significance of entropy values is evident from Figure 5 that shows the aggregate fuzzy outputs for the prediction of $C / C_{i}$ at two data points. In case 1 , which has an entropy value of 0.25 , the fuzzy output has a well-defined peak implying a lower degree of uncertainty in the prediction. In contrast, case 2 has a higher entropy value (0.54) and the output does not have a welldefined peak. The entropy values of the aggregate fuzzy outputs were calculated for predictions at all experimental

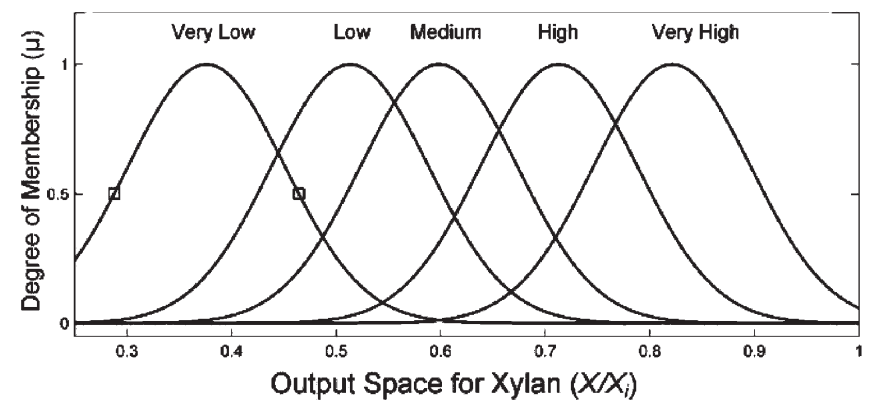

Figure 4. Membership functions for the outputs of the Mamdani-type fuzzy inference system.

data points and are reported in Table $\mathrm{V}$ along with their standard deviations. Statistical analysis did not point to any significant differences between the distributions of entropy values for lignin, cellulose, and xylan. This result is reasonable given that the entropy values quantify the uncertainty associated with the fuzzy outputs predicted by the inference system. The predictions have been made using the rule-base developed for the inference system. Hence, the entropy values represent the level of uncertainty of the knowledge source used to develop the rulebase. Given that the knowledge source for the rule-base is the same experimental data set for all three biomass components, it is reasonable that all three model outputs would have similar entropy values.

\section{Conclusions}

This study examined two different approaches to model changes in biomass composition during microwave-based alkali pretreatment of switchgrass: kinetic modeling using a time-dependent rate coefficient and a 
Table IV. Rule-base for the Mamdani-type fuzzy inference system.

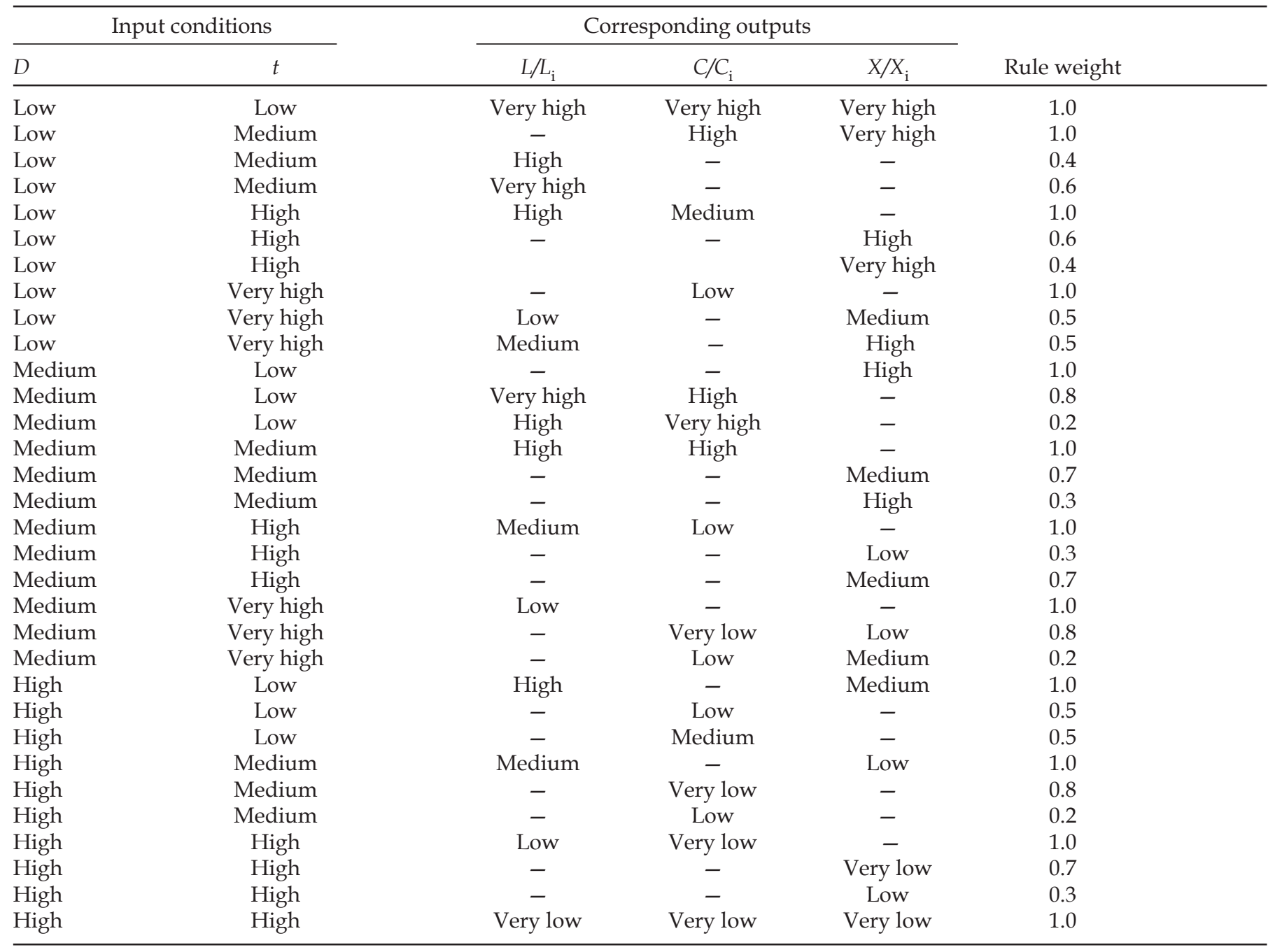

Mamdani-type fuzzy inference system. The kinetic models for lignin and xylan yielded comparable $R^{2}$ and RMSE values for both training and testing data sets and predictions had less than $2 \%$ deviations from the experimental

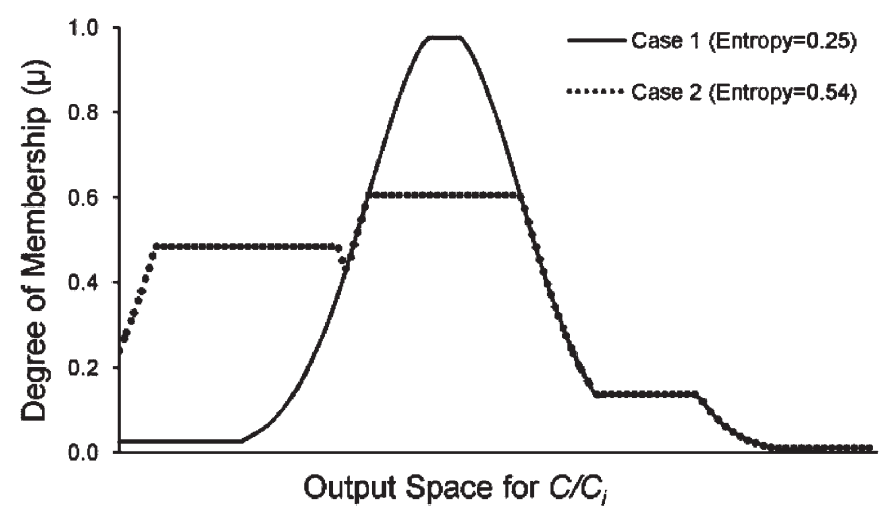

Figure 5. Aggregate fuzzy outputs of $C / C_{i}$ from the Mamdani-type inference system evaluated at two different data points indicating the relevance of entropy values. values. The kinetic model for cellulose was not as accurate, and the results had 5-7\% deviations from the experimental values. The behavior of the time-dependent rate coefficients of the kinetic model estimated from experimental data were consistent with the heterogeneity (or lack thereof) of individual biomass components. While the kinetic modeling approach is based on previously published work, the Mamdani-type fuzzy inference system was applied for the first time to model the effects of a pretreatment process. Based on the results, a distinguishing feature of the Mamdani-type inference system is the consistency in predictions using training and testing data sets for all biomass components. The predictions for lignin

Table V. Average entropy $(E)$ values for aggregate fuzzy outputs from the Mamdani-type fuzzy inference system (standard deviations are in parentheses).

\begin{tabular}{lr}
\hline Model output & Average entropy $(E)$ \\
\hline Lignin $\left(L / L_{\mathrm{i}}\right)$ & $0.353(0.101)$ \\
Cellulose $\left(C / C_{\mathrm{i}}\right)$ & $0.360(0.126)$ \\
Xylan $\left(X / X_{\mathrm{i}}\right)$ & $0.370(0.110)$ \\
\hline
\end{tabular}


were within $2 \%$ deviation from the experimental values and predictions for cellulose and xylan were within 3\% deviation from the experimental values.

While both modeling approaches were able to accurately predict changes in lignin and xylan content during the pretreatment, only the fuzzy inference system accurately predicted changes in cellulose content. It is conceivable that first-order dependence in the kinetic model is not appropriate for cellulose since crystalline and amorphous regions could be reacting differently during the pretreatment as noted by Dang and Nguyen (2006). However, the constant rate coefficient for cellulose over the course of the pretreatment seems to indicate otherwise. In addition to a better performance for cellulose, it would be easier to adapt the fuzzy inference system to account for changes in the process such as a different biomass or different alkali or the addition of an output such as changes in cellulose crystallinity. As long as some qualitative knowledge about these changes is available, the membership functions and rule-base can be manually modified and limited experimental data would suffice for validation. However, adapting the kinetic model to such changes would necessitate new experimental data to estimate the model parameters in addition to validation data.

Changes in biomass composition have economic consequences on bioethanol production from lignocellulosic materials as they directly impact the amount of lignin available for recovery as a boiler-fuel and the amount of carbohydrates available for hydrolysis and fermentation into ethanol. Hence, the ability of these models to predict changes in biomass composition makes them useful tools for process simulations and economic assessments of bioethanol production from lignocellulosic materials.

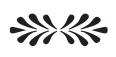

The authors would like to thank the Golden LEAF Foundation of North Carolina and the North Carolina Biotechnology Center for their funding support and Novozymes North America Inc. (Franklinton, NC) for their assistance with HPLC analysis.

\section{References}

Avrami M. 1940. Kinetics of phase change II. J Chem Phys 8:212-224.

Babuska R. 1998. Fuzzy modeling for control. Boston, MA: Kluwer Academic Publishers.

Bamford CH, Tipper CFH. 1980. Reactions in the solid state. In: Compton RG, Hancock G, editors. Chemical kinetics. New York, NY: Elsevier, p 13-101.

Bogren J, Brelid H, Theliander H. 2007. Reaction kinetics of softwood kraft delignification - General considerations and experimental data. Nord Pulp Pap Res J 22:177-183.

Chiang VL, Yu J, Eckert RC. 1990. Isothermal reaction kinetics of kraft delignification of Douglas-fir. J Wood Chem Technol 10:293-310.
Chum HL, Johnson DK, Black SK, Overend RP. 1990. Pretreatment catalyst effects and the combined severity parameter. Appl Biochem Biotechnol 24-5:1-14.

Conner AH. 1984. Kinetic modeling of hardwood prehydrolysis. 1. Xylan removal by water prehydrolysis. Wood Fiber Sci 16:268-277.

Conner AH, Wood BF, Hill CGJ, Harris JF. 1985. Kinetic model for the dilute sulfuric acid saccharification of lignocellulose. J Wood Chem Technol 5:461-489.

Converse AO, Ooshima H, Burns DS. 1990. Kinetics of enzymatic hydrolysis of lignocellulosic materials based on surface area of cellulose accessible to enzyme and enzyme adsorption on lignin and cellulose. Appl Biochem Biotechnol 24-5:67-73.

Dang V, Nguyen KL. 2006. Characterisation of the heterogeneous alkaline pulping kinetics of hemp woody core. Bioresour Technol 97:1353-1359.

Dang VQ, Nguyen KL. 2007. A universal kinetic equation for characterising the fractal nature of delignification of lignocellulosic materials. Cellulose 14:153-160.

Dunn CP, Stearns F, Guntenspergen GR, Sharpe DM. 1993. Ecological benefits of the Conservation Reserve Program. Conserv Biol 7:132-139.

Gustafson RR, Slelcher CA, Mckean WT, Finlayson BA. 1983. Theoretical model of the kraft pulping process. Ind Eng Chem Proc Des Dev 22:87-96.

$\mathrm{Hu}$ ZH, Wen ZY. 2008. Enhancing enzymatic digestibility of switchgrass by microwave-assisted alkali pretreatment. Biochem Eng J 38:369-378.

Hung WL. 2003. A note on entropy of intuitionistic fuzzy sets. Int J Uncertain Fuzziness Knowledge-Based Syst 11:627-633.

Jacobsen SE, Wyman CE. 2000. Cellulose and hemicellulose hydrolysis models for application to current and novel pretreatment processes. Appl Biochem Biotechnol 84-6:81-96.

Kerr AJ. 1970. Kinetics of kraft pulping-Progress in development of a mathematical model. Appita 24:180-188.

Keshwani DR, Cheng JJ. 2008. Modeling of microwave based chemical pretreatment of switchgrass. 2008 ASABE Annual Meeting Paper No. 083954, Providence, RI.

Keshwani DR, Cheng JJ. 2009. Switchgrass for bioethanol and other valueadded applications: A review. Bioresour Technol 100:1515-1523.

Keshwani DR, Jones DD, Brand RM. 2005. Takagi-Sugeno fuzzy modeling of skin permeability. Cutan Ocul Toxicol 24:149-163.

Keshwani DR, Cheng JJ, Burns JC, Li L, Chiang V. 2007. Microwave pretreatment of switchgrass to enhance enzymatic hydrolysis. 2007 ASABE Annual Meeting Paper No. 077127, Minneapolis, MN.

Keshwani DR, Jones DD, Meyer GE, Brand RM. 2008. Rulebased Mamdani- type fuzzy modeling of skin permeability. Appl Soft Comput 8: 285-294.

Kumar P, Coronel P, Simunovic J, Truong VD, Sandeep KP. 2007. Measurement of dielectric properties of pumpable food materials under static and continuous flow conditions. J Food Sci 72:E177-E183.

Lee D, Yu AHC, Wong KKY, Saddler JN. 1994. Evaluation of the enzymatic susceptibility of cellulosic substrates using 
specific hydrolysis rates and enzyme adsorption. Appl Biochem Biotechnol 45-6:407-415.

McLaughlin SB. 1993. New switchgrass biofuels research program for the southeast. P 1992 Ann Aut Techn, pp. 111-115.

Montane D, Salvado J, Farriol X, Jollez P, Chornet E. 1994. Phenomenological kinetics of wood delignification-Application of a timedependent rate-constant and a generalized severity parameter to pulping and correlation of pulp properties. Wood Sci Technol 28:387-402.

Mosier N, Wyman C, Dale B, Elander R, Lee YY, Holtzapple M, Ladisch M. 2005. Features of promising technologies for pretreatment of lignocellulosic biomass. Bioresour Technol 96:673-686.

Nelson SO, Datta CJ. 2001. Dielectric properties of food materials and electric field interactions. In: Datta AK, Anantheswaran RC, editors. Handbook of microwave technology for food applications. New York: Marcel Dekker Inc., p 69-107.

Overend RP, Chornet E. 1987. Fractionation of lignocellulosics by steamaqueous pretreatments. Philos Trans R Soc Lond A 321:523-536.

Pannier AK, Brand RM, Jones DD. 2003. Fuzzy modeling of skin permeability coefficients. Pharm Res 20:143-148.

Parrish DJ, Fike JH. 2005. The biology and agronomy of switchgrass for biofuels. Crit Rev Plant Sci 24:423-459.

Ramos LP. 2003. The chemistry involved in the steam treatment of lignocellulosic materials. Quim Nova 26:863-871.

Ross TJ. 1995. Fuzzy logic with engineering applications. New York, NY: McGraw-Hill, Inc.

Saeman JF. 1945. Kinetics of wood saccharification-Hydrolysis of cellulose and decomposition of sugars in dilute acid at high temperature. Ind Eng Chem Res 37:43-52.

Samhouri M, Abu-Ghoush M, Yaseen E, Herald T. 2009. Fuzzy clusteringbased modeling of surface interactions and emulsions of selected whey protein concentrate combined to iota-carrageenan and gum arabic solutions. J Food Eng 91:10-17.

Sanderson MA, Reed RL, McLaughlin SB, Wullschleger SD, Conger BV, Parrish DJ, Wolf DD, Taliaferro C, Hopkins AA, Ocumpaugh WR, Hussey MA, Read JC, Tischler CR. 1996. Switchgrass as a sustainable bioenergy crop. Bioresour Technol 56:83-93.

Shapiro SS, Wilk MB. 1965. An analysis of variance test for normality (complete samples). Biometrika 52:591-611.

Silverstein RA, Chen Y, Sharma-Shivappa RR, Boyette MD, Osborne J. 2007. A comparison of chemical pretreatment methods for improving saccharification of cotton stalks. Bioresour Technol 98:3000- 3011.

Sluiter A, Hames B, Ruiz R, Scarlata C, Sluiter J, Templeton D. 2007. Determination of structural carbohydrates and lignin in biomass. Laboratory Analytical Procedure, NREL/ TP-510-42618. Golden, CO: National Renewable Energy Laboratory.

Sridar V. 1998. Microwave radiation as a catalyst for chemical reactions. Curr Sci 74:446-450.

Sun Y, Cheng JY. 2002. Hydrolysis of lignocellulosic materials for ethanol production: A review. Bioresour Technol 83:1-11.

Thomason WE, Raun WR, Johnson GV, Taliaferro CM, Freeman KW, Wynn KJ, Mullen RW. 2004. Switchgrass response to harvest frequency and time and rate of applied nitrogen. J Plant Nutr 27:1199-1226.

Torbert HA, Krueger E, Kurtener D. 2008. Soil quality assessment using fuzzy modeling. Int Agrophys 22:365-370.

Turanyi T, Rabitz H. 2000. Local methods. In: Saltelli A, Chan K, Scott EM, editors. Sensitivity analysis. Chichester, New York: Wiley, p 81-99.

Zhu SD, Yu ZN, Wu YX, Zhang X, Li H, Gao M. 2005. Enhancing enzymatic hydrolysis of rice straw by microwave pretreatment. Chem Eng Commun 192:1559-1566. 